\title{
Bacteriemia primaria por Listeria monocytogenes en paciente con cirrosis hepática. Caso clínico
}

\author{
Leonardo Chanqueo $C^{1,3}$, Catalina G utiérrez $R^{a}$, \\ Rodolfo Armas $\mathrm{M}^{2}$, G abriela U rriola J3b, \\ Marisol Bustos $\mathbf{M}^{1}$, Cecilia Tapia $\mathrm{P}^{3}$, Patricia Vásquez $\mathrm{T}^{1}$. \\ Primary Listeria monocytogenes \\ infection in a cirrhotic woman. \\ Report of one case
}

L monocytogenes infections are infrequent. Sepsis in pregnant women and newborns and central nervous system infections in the elderly are the most common clinical manifestations. We report a 61 years old woman with diabetes Mellitus and a Child B hepatic cirrhosis, admitted for persistent fever. Blood cultures were positive for Listeria monocytogenes. Cerebrospinal fluid was normal and sterile. She was treated with ampicillin and amikacin with a good response. Control blood cultures were negative. She was discharged 14 days after in good conditions (Rev Méd Chile 2008; 136: 225-9).

(Key words: Amikacin; Listeria monocytogenes; Liver cirrhosis)

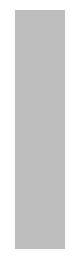

Recibido el 5 de marzo, 2007. Aceptado el 10 de julio, 2007.

Este trabajo no tuvo fuente de apoyo financiero.

${ }^{1}$ Unidad de Infectología, Servicio de Medicina Interna, Hospital San Juan de Dios. ${ }^{2}$ Unidad de Gastroenterología, Departamento de Medicina Interna Occidente, Facultad de Medicina, Universidad de Chile. ${ }^{3}$ Laboratorio de Microbiología, Hospital San Juan de Dios.

a Becaria de Medicina Interna, Facultad de Medicina Occidente, Universidad de Chile

becnólogo Médico

T as infecciones por L monocytogenes y su _aislamiento desde muestras clínicas son infrecuentes. Sin embargo, cuando la infección se produce son cuadros severos con alta mortalidad en población de riesgo ${ }^{1,2}$.

El género Listeria spp. corresponde a un grupo de bacterias gram positivas, anaerobias facultativas, de disposición cocobacilar, poseen un flagelo polar, no tienen cápsula ni forman esporas. Existen 6 especies: L monocytogenes, L seeligeri, L welshi-

Correspondencia a: Dr. Leonardo Chanqueo C. Av. Portales 3225. Santiago, Chile. Fono: 5625742283.

E mail: lchanqueo@gmail.com meri, L innocua, L ivanovii y L grayi, sin embargo sólo L monocytogenes es patogénica en humanos. Se encuentra diseminada ampliamente en el ambiente, incluyendo tierra, agua, alimentos y en deposiciones humanas y de animales ${ }^{3,4}$.

Las manifestaciones clínicas son variadas, siendo las más conocidas la sepsis en mujeres embarazadas, neonatos y la infección del sistema nervioso central en ancianos ${ }^{5,6}$. En los últimos años existen numerosos reportes de otras manifestaciones sistémicas de infección por L monocytogenes especialmente en pacientes inmunodeprimidos ${ }^{7,8}$.

Comunicamos el caso de una paciente portadora de cirrosis hepática y de diabetes mellitus 
que presentó una bacteriemia primaria por L monocytogenes.

\section{Caso CLÍNICO}

Paciente de sexo femenino de 61 años de edad, con antecedentes de diabetes mellitus tipo 2, hipertensión arterial y cirrosis hepática Child B de etiología no precisada, en control en policlínico de gastroenterología.

Consultó por cuadro de una semana de evolución caracterizado por fiebre de predominio nocturno, cuantificada hasta en $39^{\circ} \mathrm{C}$ y precedida de un día de diarrea no disentérica y autolimitada. Se evaluó en Servicio de Urgencia, se realizaron exámenes dentro de los cuales destacan: hemograma sin leucocitosis ni desviación a izquierda, pruebas de coagulación, electrolitos plasmáticos y función renal normales. Se realizó ecotomografía abdominal que mostró esplenomegalia, signos de daño hepático crónico y enfermedad diverticular del colon. No se consignó fiebre y se decidió alta sin terapia medicamentosa específica.

Dada la persistencia de fiebre por más de una semana desde la primera consulta, se hospitalizó para estudio. La paciente negó sintomatología respiratoria, digestiva y urinaria, no tuvo otros episodios de diarrea. En el examen físico se constató febril hasta $38,7^{\circ} \mathrm{C}$, presión arterial 140/80, frecuencia cardiaca 108 por min, piel y mucosas hidratadas, sin adenopatías palpables, no se observó telangiectasias, ictericia ni lesiones cutáneas.
Examen cardiopulmonar normal; abdomen globuloso, blando, depresible e indoloro, sin ascitis ni circulación colateral. Resto del examen normal.

El estudio realizado durante la hospitalización incluyó: hemocultivos automatizados (Bact Alert ${ }^{\circledR}$ ), urocultivo, exámenes inmunológicos y de imágenes.

De los exámenes de laboratorio inmunológico destacó: anticuerpos antinucleares (ELSA binding-site) negativos; complemento C3: $86,3 \mathrm{mg} / \mathrm{dL}$ (VR: 90-180), C4: 17,3 mg/dL (VR: 10-40) y factor reumatoideo (aglutinación en placa) negativo. En la radiografía de tórax AP se apreció silueta cardiaca de tamaño normal, campos pulmonares simétricos, sin focos de condensación (Figura 1a).

Evolucionó en regulares condiciones generales, con fiebre persistente y cefalea holocraneana de intensidad leve a moderada tipo opresiva- pulsátil y dolor retroesternal opresivo leve asociado a bradicardia e hipotensión leve. El electrocardiograma mostró bradicardia sinusal, segmento ST normal, las enzimas cardiacas fueron normales. La ecocardiografía transtorácica reveló hipertrofia septal leve y presencia de derrame pericárdico de grado leve a moderado; la radiografía de tórax mostró aumento del tamaño de la silueta cardiaca y derrame pleural (Figura 1b).

Los hemocultivos (2) fueron positivos a las 20 y 21 $\mathrm{h}$ de incubación, se informó en la tinción de gram: bacilos gram $(+)$ cortos, no esporulados. Se traspasó a medios de cultivo: agar sangre cordero (Biomeneux ${ }^{\circledR}$ ), agar chocolate $\left(\right.$ Biomerieux ${ }^{\circledR}$ ) y caldo cerebro corazón, creciendo colonias pequeñas, translúcidas, grises, catalasa (+), con presencia de beta hemólisis, cuya tinción de gram demostró bacilos

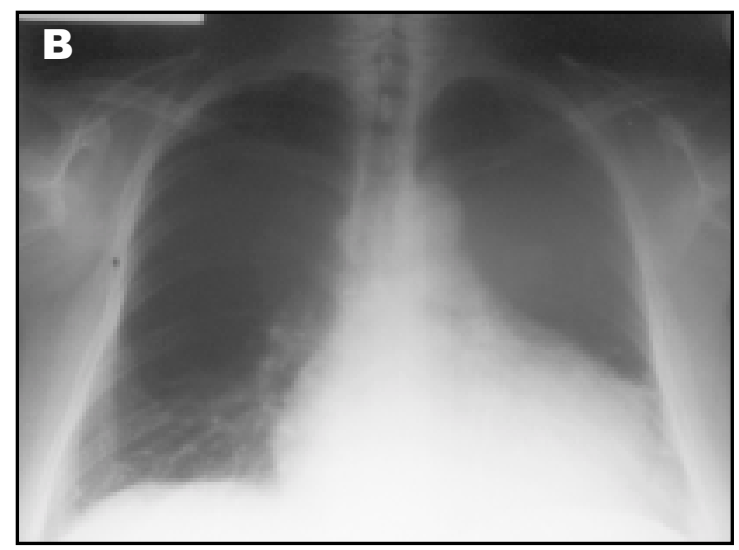

Figura 1. En las imágenes se observa la evolución de la radiografía de tórax al ingreso (a) y una semana después (b), en esta última hay presencia de ensanchamiento de silueta cardiaca y derrame pleural. 
gram $(+)$, cocobacilos a difteroides cortos, delgados, no esponulados (Figuras 2a y 2b). El desarmollo fue a $4^{\circ} \mathrm{C}$, con movilidad a $20^{\circ} \mathrm{C}$ en tumbos y a $25^{\circ} \mathrm{C}$ en paraguas, de las pruebas bioquímicas clásicas: esculina (+), glucosa (+), hidrólisis de hipurato de sodio (+) y H2S (-). La identificación bioquímica por sistema automatizado Vitek ${ }^{\circledR}$ (Biomerieux ${ }^{\circledR}$ ) fue de Listeria spp., que fue confirmada por Instituto de Salud Pública como L monocytogenes. La susceptibilidad antimicrobiana realizada en equipo automatizado resultó sensible a ampicilina, gentamicina y trimetroprin-sulfametoxazol.

Se realizó punción lumbar, obteniendo líquido cefalorraquídeo sin dificultad, el que fue incoloro, de aspecto claro, sin leucocitos, glucosa $76 \mathrm{mg} /$ $\mathrm{dL}$, proteínas totales $0,05 \mathrm{~g} / \mathrm{L}$, ADA $5 \mathrm{U} / \mathrm{L}$, la tinción de gran y cultivo corriente aeróbico a las $72 \mathrm{~h}$ de incubación fueron negativos.

Se inició tratamiento antimicrobiano, inmediatamente después de la punción lumbar y conociendo el resultado de la tinción de gram de hemocultivos, con ampicilina $1 \mathrm{~g}$ cada $6 \mathrm{~h}$ más amikacina $600 \mathrm{mg} /$ día; el urocultivo resultó negativo.

Evolucionó con febrículas durante las primeras $72 \mathrm{~h}$ de tratamiento por lo que se aumentó la dosis de ampicilina a $2 \mathrm{~g}$ cada $6 \mathrm{~h}$ y se suspendió la amikacina al descartar compromiso de SNC. Posteriormente continuó afebril y disminuyeron los parámetros inflamatorios.

Otros exámenes que se realizaron: anticuerpos anti VIH (ELISA), antígeno de superficie hepatitis $\mathrm{B}$ y anticuerpos antivirus hepatitis $\mathrm{C}$ que resultaron negativos; alfafetoproteína 3,62 UI/mL (VR: 0-

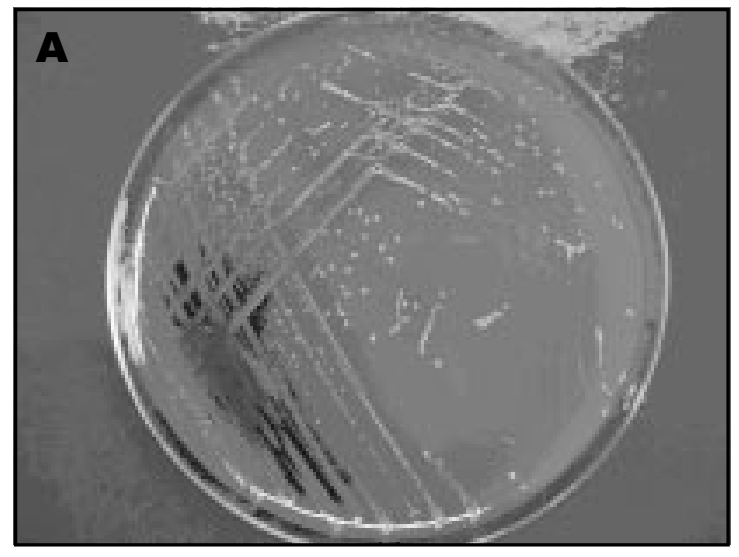

5,81), electroforesis de proteínas con hipoalbuminemia e hipergamaglobulinemia policlonal, no se realizó ferritina sérica.

En la radiografía de tórax de control se observó leve aumento del derrame pleural, se realizó toracocentesis diagnóstica que dio salida a líquido cetrino, con recuento de leucocitos 600 $\mathrm{cel} / \mathrm{mm}^{3}, 61 \%$ mononucleares y $39 \%$ polimorfonucleares, glucosa $181 \mathrm{mg} / \mathrm{dL}$, colesterol $<7 \mathrm{mg} / \mathrm{dL}$, proteínas totales 0,6 g/L, LDH $42 \mathrm{U} / \mathrm{L}, \mathrm{ADA} 3 \mathrm{U} / \mathrm{L}$, $\mathrm{pH} 7,56$, la tinción de gram y cultivo corriente aeróbico fueron negativos.

Se realizó tomografía axial computarizada de abdomen y pelvis para descartar focos intraabdominales, que mostró signos de daño hepático crónico con esplenomegalia, moderada cantidad de líquido libre, sin colecciones. La ecocardiografía transtorácica demostró disminución del derrame pericárdico.

Luego de completar 14 días de tratamiento intravenoso se decidió dar el alta hospitalaria, continuando terapia con ampicilina v.o. por 7 días. La evolución posterior fue favorable desde el punto de vista clínico y de laboratorio con hemocultivos de control negativos.

\section{Discusión}

La listeriosis es una zoonosis que puede presentarse esporádicamente 0 en epidemias y el mecanismo de transmisión es fundamentalmente el consumo de alimentos contaminados ${ }^{9,10}$. Una vez

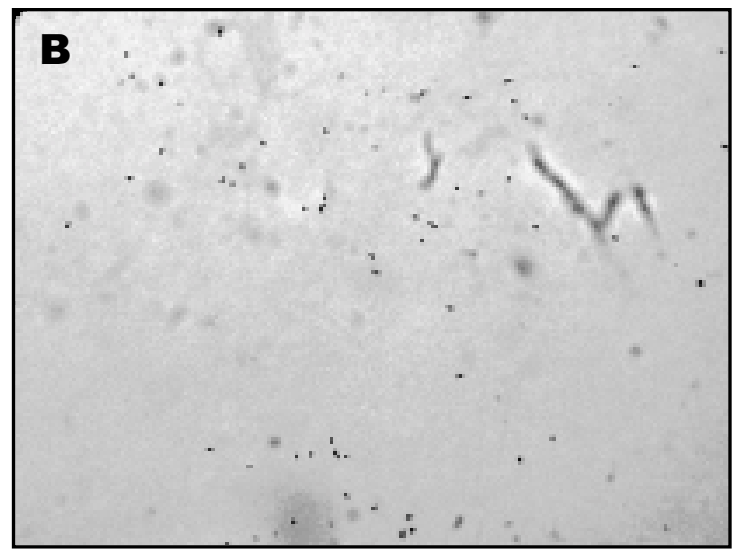

Figuras 2. Imagen de la colonia bacteriana en agar sangre (a) y tinción de gram de la colonia (b) de L monocytogenes desarrollada del frasco de hemocultivos. 
ingerida la L monocytogenes, dependiendo de la inmunidad del huésped y posiblemente el tamaño del inóculo, cruza la mucosa intestinal y llega al torrente sanguíneo. Desde aquí, puede diseminarse a cualquier órgano, sin embargo tiene clara predilección por el sistema nervioso central y placenta. Por este motivo, las infecciones por L monocytogenes más conocidas son las infecciones del SNC, primariamente meningitis y encefalitis ${ }^{20}$; las infecciones en embarazadas, en quienes provoca bacteriemia, infecciones placentarias con corioamnionitis e infecciones fetales que pueden llevar a abortos y partos prematuros ${ }^{4}$, y la bacteriemia sin foco clínico.

Otras manifestaciones menos frecuentes son la endocarditis, miocarditis, linfadenitis, neumonías, hepatitis, colecistitis, peritonitis, abscesos localizados y empiema, endoftalmitis, etc ${ }^{1}$.

Especialmente susceptibles son los pacientes ancianos $^{12}$, aquellos enfermos con trastornos de la inmunidad celular, como son los trasplantados, aquellos con infección por VIH/SIDA, terapia inmunosupresora, los portadores de neoplasias, alcoholismo crónico y cirrosis. Estudios españoles ${ }^{1,13}$, donde tienen la más alta prevalencia de infección por L monocytogenes en la población general, describen como importantes factores predisponentes la diabetes mellitus $(25 \%$ de los casos) y la cirrosis hepática, ambas condiciones presentes en nuestra paciente.

En individuos sanos la infección por L monocytogenes suele provocar cuadros de diarrea febril autolimitada o portación asintomática ${ }^{10,15}$, sin embargo en individuos inmunosuprimidos puede llevar a cuadros de listeriosis invasora.

En el caso que comunicamos, probablemente la puerta de entrada de la infección fue a partir de la ingesta de alimentos contaminados y diseminación secundaria con bacteriemia, esto por el antecedente de cuadro diarreico autolimitado. Sin embargo, no pudo ser aclarado en la anamnesis el consumo de algún alimento sospechoso.

La paciente tenía el antecedente de cirrosis hepática de años de evolución como factor predisponerte evidente, no se demostró otra enfermedad inmunosupresora con el estudio realizado, como: enfermedad del colágeno activa, sobreinfección por virus hepatitis B o C, infección por VIH/SIDA. No demostramos diseminación a SNC, ni pleural, no realizamos estudio del líquido ascítico, no había ascitis en el examen inicial y el estudio imagenológico posterior en que se demostró ascitis, la paciente clínicamente evolucionó satisfactoriamente.

La infección por L monocytogenes en cirróticos es fundamentalmente por trastornos en los mecanismos del sistema inmune como son la disfunción del sistema retículo-endotelial, disminución del complemento y de la actividad macrofági$\mathrm{ca}^{1,16}$. Incluso puede existir peritonitis bacteriana espontánea (PBE), aunque es raro ${ }^{17,18}$.

Hubo desarrollo de ascitis, derrame pleural y pericárdico durante la hospitalización, lo cual puede ser explicado por diversos mecanismos, como son la descompensación de cirrosis hepática en contexto de infección, desnutrición proteica con albúmina baja, alteraciones inmunológicas secundarias a la infección y no necesariamente como infección diseminada, esto porque el compromiso multisistémico dado por L monocytogenes es severo y de evolución más tórpida. La pericarditis por L monocytogenes es un cuadro poco frecuente descrito en la literatura, se presenta habitualmente en el contexto de una bacteriemia debida a este microorganismo y se asocia a endocarditis infecciosa y abscesos intracavitarios $^{19}$. En nuestro caso, si bien se pesquisó en el curso de la infección la presencia de derrame pericárdico, éste fue asintomático, sin signos de taponamiento y se resolvió con el uso de terapia antibiótica; la ecocardiografía transtorácica descartó otras complicaciones.

L monocytogenes es sensible in vitro a un grupo de antibióticos, incluidos penicilina, eritromicina, ampicilina, cotrimoxazol, cloranfenicol, tetraciclina y aminoglucósidos; actualmente se utiliza ampicilina sola o asociada a aminoglucósidos y cotrimoxazol en los casos de alergia a penicilina y derivados. En relación al tiempo de tratamiento antimicrobiano en los casos de bacteriemia sin foco, la duración no está claramente documentada y en general se recomienda por un período de al menos dos a tres semanas, lo cual ha disminuido las recaídas particularmente en pacientes inmunocomprometidos ${ }^{20}$.

La mortalidad en distintas series oscila entre $28 \%$ hasta $61 \% 1,13,14$, siendo mayores las tasas de mortalidad en ancianos, infección del sistema nervioso central y otras patologías crónicas avanzadas. 
Probablemente la incidencia de esta infección aumente en los próximos años en nuestro país, en relación al progresivo envejecimiento de la población, aumento del número y mayor esperanza de vida de pacientes inmunodeprimidos. Por tanto es importante incorporar este microorganismo como

\section{REFERENCIAS}

1. JuLía A, Jiménez A, De Górgolas M, Fernández $R$, FERNÁnDEZ ML. Infecciones por Listeria monocytogenes en el adulto. Aspectos clínicos y microbiológicos de una enfermedad cambiante. Enferm Infecc Microbiol Clin 2001; 19: 297-303.

2. Siegman-igra Y, Levin R, Weinberger M, Golan Y, Schwartz D, Samra Z, Konigsberger H et al. Listeria monocytogenes Infection in Israel and Review of Cases Worldwide. Emerg Infect Dis 2002; 8: 305-10.

3. Biwe J, Rocourt J, Swaminathan B. Listeria and Erysipelothrix. En: Murray P.R., Baron E.J., Jorgensen J.H, Phaller M.A., Yolken R.H. (ed). Manual of Clinical Microbiology, ${ }^{\text {th }}$ ed. American Society of Microbiology. Washington DC. 2003; 461-6468.

4. Vázquez-boland J, Kuhn M, Berche P, Chakraborty T, Dominguez-bernal G, Goebel W et al. Listeria Pathogenesis and Molecular Virulence Determinants. Clin Microbiol Rev 2001; 14: 584-640.

5. Lobos T, Silva E, Maldonado A, Valenzuela M, Arancibia E, Montecinos D. Listeriosis perinatal. Rev Chil Infectol 1984; 1: 101-3.

6. Bravo M, FerRer S, TrujiLO S. Romboencefalitis por Listeria monocytogenes. Caso clínico. Rev Méd Chile 1998; 126: 828-32.

7. Rivero G, Torres H, Rolston K, Kontoyiannis D. Listeria monocytogenes infection in patients with cancer. Diagn Microbiol Infect Dis 2003; 47: 393-8.

8. Guerra J, Muinelo I, Pérez-simón M, Bianco B, Carro J, Alcoba M. Listeriosis en pacientes con infección por el virus de la inmunodeficiencia humana en España. Aportación de tres casos y revisión de la literatura. Enferm Infecc Microbiol Clin 2004; 22: 18-21.

9. Crespo M, Vélez J, Castañeda C, Hoyos F, Ligia M, SalazAR J. Aislamiento de Listeria monocytogenes en un hospital de tercer nivel. Colombia Med 1999; 30: 89-98. agente etiológico en el diagnóstico diferencial de infecciones en inmunodeprimidos, como también en brotes de infecciones asociadas a alimentos contaminados. En este sentido es fundamental la educación, en relación al consumo de productos crudos, no pasteurizados o en lugares no habilitados.

10. Aurel P, Fiorucci G, Caroll D, Marchiaro G, Novara O, Leone L, Salmaso S. An Outbreak of Febrile Gastroenteritis Associated with Corn Contaminated by Listeria monocytogenes. N Engl J Med 2000; 342: 1236-41.

11. Iluanes S, Araya P, Ferrer S, Cartier L, Bravo M, CASTILO S. Romboencefalitis: una forma de infección por Listeria monocytogenes en el sistema nervioso central. Rev Méd Chile 2003; 131: 921-8.

12. Remacha MA, HerRera JA, Esteban A, Roiz V, Quiroga L, PARRA I. Bacteriemia por Listeria monocytogenes. Rev Diagn Biol 2002; 51: 111-2.

13. Arias Miranda I, Nuño F, MenÉndez J, Fonseca E, CALderón M. Listeriosis en el adulto: Revisión de 10 casos. An Med Interna (Madrid) 2004; 21: 31-6.

14. Gerner-Smidt P, Ethelberg S, Schielerup P, ChristenSEN J, ENgBerg J, Fussing V ET AL. Invasive listeriosis in Denmark 1994-2003: a review of 299 cases with special emphasis on risk factors for mortality. Clin Microbiol Infect 2005; 11: 618-24.

15. OoI S, Sorber B. Gastroenteritis due to Listeria monocytogenes. Clin Infect Dis 2005; 40: 1327-32.

16. Fica A. Diagnóstico, manejo y prevención de infecciones en pacientes con cirrosis hepática. Rev Chil Infect 2005; 22: 63-74.

17. Nolia-Salas J, Almela M, Gasser I, Latorre C, Salvado M, Col P. Spontaneous Listeria monocytogenes peritonitis: a population-based study of 13 cases collected in Spain. Am J Gastroenterol 2002; 97: 1507-11.

18. Espinoza-Gómez F, Newton-Sánchez O, Melnikov V, PINZÓN L. Peritonitis espontánea por Listeria monocytogenes, en un paciente con cirrosis hepática: Caso clínico. Rev Méd Chile 2006; 134: 1171-4.

19. Manso C, Rivas I, Peraire J, Vidal F, Richart C. Fatal Listeria meningitis, endocarditis and pericarditis in a patient with Haemochromatosis. Scand J Infect Dis 1997; 29: 308-9.

20. Hof H, Nichteriein T, KRetschmar M. Management of Listeriosis. Clin Microbiol Rev 1997; 10: 345-57. 